\title{
Three-Dimensional Mapping of Hippocampal Anatomy in Unmedicated and Lithium-Treated Patients with Bipolar Disorder
}

\author{
Carrie E Bearden*,', Paul M Thompson², Rebecca A Dutton², Benício N Frey ${ }^{3,4}$, Marco AM Peluso ${ }^{3,5}$, \\ Mark Nicoletti ${ }^{6}$, Nicole Dierschke ${ }^{3}$, Kiralee M Hayashi ${ }^{2}$, Andrea D Klunder ${ }^{2}$, David C Glahn ${ }^{3}$, \\ Paolo Brambilla ${ }^{7,8}$, Roberto B Sassi ${ }^{5,9}$, Alan G Mallinger ${ }^{10}$ and Jair C Soares ${ }^{6}$ \\ 'Department of Psychiatry \& Biobehavioral Sciences, Semel Institute for Neuroscience and Human Behavior, University of California, Los Angeles, \\ CA, USA; ${ }^{2}$ Laboratory of Neurolmaging, Department of Neurology, University of California, Los Angeles, CA, USA; ${ }^{3}$ Department of Psychiatry, \\ University of Texas Health Science Center at San Antonio, San Antonio, TX, USA; ${ }^{4}$ Department of Biochemistry and Bipolar Disorders Program, \\ Federal University of Rio Grande do Sul, Porto Alegre, Brazil; ${ }^{5}$ Department of Psychiatry, University of Sao Paulo School of Medicine, Sao Paulo, \\ Brazil; ' Department of Psychiatry, UNC Chapel Hill School of Medicine, Chapel Hill, NC, USA; ${ }^{7}$ Scientific Institute IRCCS, E. Medea, Udine, Italy; \\ ${ }^{8}$ Section of Psychiatry, Department of Pathology and Experimental \& Clinical Medicine, University of Udine, Udine, Italy; ${ }^{9}$ Department of \\ Psychiatry, Massachusetts General Hospital, Harvard University, Boston, MA, USA; ${ }^{10}$ Department of Psychiatry, University of Pittsburgh School of \\ Medicine, Pittsburgh, PA, USA
}

\begin{abstract}
Declarative memory impairments are common in patients with bipolar illness, suggesting underlying hippocampal pathology. However, hippocampal volume deficits are rarely observed in bipolar disorder. Here we used surface-based anatomic mapping to examine hippocampal anatomy in bipolar patients treated with lithium relative to matched control subjects and unmedicated patients with bipolar disorder. High-resolution brain magnetic resonance images were acquired from 33 patients with bipolar disorder (2I treated with lithium and 12 unmedicated), and 62 demographically matched healthy control subjects. Three-dimensional parametric mesh models were created from manual tracings of the hippocampal formation. Total hippocampal volume was significantly larger in lithium-treated bipolar patients compared with healthy controls (by 10.3\%; $p=0.00 \mathrm{I}$ ) and unmedicated bipolar patients (by 13.9\%; $p=0.003$ ). Statistical mapping results, confirmed by permutation testing, revealed localized deficits in the right hippocampus, in regions corresponding primarily to cornu ammonis I subfields, in unmedicated bipolar patients, as compared to both normal controls $(p=0.0 \mathrm{I})$, and in lithium-treated bipolar patients $(p=0.03)$. These findings demonstrate the sensitivity of these anatomic mapping methods for detecting subtle alterations in hippocampal structure in bipolar disorder. The observed reduction in subregions of the hippocampus in unmedicated bipolar patients suggests a possible neural correlate for memory deficits frequently reported in this illness. Moreover, increased hippocampal volume in lithium-treated bipolar patients may reflect postulated neurotrophic effects of this agent, a possibility warranting further study in longitudinal investigations.

Neuropsychopharmacology (2008) 33, 1229-1238; doi:I0. I038/sj.npp. I 301507; published online 8 August 2007
\end{abstract}

Keywords: mood disorder; magnetic resonance imaging; hippocampus; medication effects; neurotrophins; brain mapping

\section{INTRODUCTION}

The hippocampus is essential for the formation of new autobiographical and factual memories (Eichenbaum and

This work presented in part as an oral presentation at the 36th Annual Meeting of the Society for Neuroscience, Atlanta, GA, October 14-18 2006.

*Correspondence: Dr CE Bearden, Department of Psychiatry \& Biobehavioral Sciences, University of California, Los Angeles, 300 Building Medical Plaza, Suite 2265, Los Angeles, CA 90095, USA, Tel: + I 310206 2983, Fax: + I 3107949517,

E-mail: cbearden@mednet.ucla.edu

Received 20 December 2006; revised 31 May 2007; accepted 8 June 2007
Fortin, 2005). Consistent findings of declarative memory deficits in patients with bipolar disorder (Altshuler et al, 2004; Bearden et al, 2006; Gourovitch et al, 1999; Kieseppa et al, 2005; van Gorp et al, 1999) suggest the presence of hippocampal pathology; in addition, there is increasing evidence that this brain structure also plays a role in the modulation of emotion (Kempermann, 2002). However, most studies have not identified hippocampal volume deficits in bipolar disorder (see Brambilla et al, 2005 for a review). A recent meta-analysis (McDonald et al, 2004) found that, of the eight studies that had examined hippocampal volume in bipolar disorder (a combined total of 245 patients and 273 controls), hippocampal volumes did not significantly differ between patients and controls. 
Significant heterogeneity among studies was detected as to whether differences were found in both the left and right hippocampus-amygdala complex. The authors concluded that one of the most striking findings was the volume preservation of most brain regions studied, particularly temporal lobe structures such as the hippocampus. This absence of deficit is surprising, given that hippocampal volume deficits have been demonstrated by previous metaanalyses to be a consistent feature of both schizophrenia (Wright et al, 2000) and unipolar depression (Videbech and Ravnkilde, 2004).

However, the extensive heterogeneity detected indicates the need for further studies, with new methods, to determine whether localized hippocampal alterations may exist in specific clinical subgroups of bipolar illness. Conventional studies of global hippocampal volume cannot examine whether the hippocampal formation is unevenly affected in bipolar illness, as the hippocampus is a highly differentiated structure, and localized deficits may be present in specific subregions of the hippocampus.

In addition, some psychotropic medications commonly used to treat bipolar disorder may reverse or attenuate primary neuroanatomic abnormalities associated with the illness (McDonald et al, 2004). For example, lithium may have neurotrophic properties, leading to increased gray matter volume in vivo, in the whole brain (Moore et al, 2000b; Sassi et al, 2002) and the anterior cingulate (Sassi et al, 2004). Magnetic resonance spectroscopy has also revealed increases in cortical $\mathrm{N}$-acetyl-aspartate, a putative marker of neuronal integrity, in both bipolar patients and controls following lithium administration (Moore et al, 2000a; Silverstone et al, 2003). However, no study to date has examined regionally specific effects of lithium and whether it may be associated with alterations in hippocampal structure in bipolar patients.

Here we report results of the first study, to our knowledge, to use three-dimensional (3-D)surface mesh models to evaluate the structural integrity of the hippocampus in patients with bipolar disorder. This technique (Thompson et al, 2004a) improves upon other methods in that it visualizes the spatial profile of neuropathological abnormalities, allowing more refined neuroanatomic localization if regionally specific alterations are present in bipolar patients. The mapping approach has been used to detect subtle or localized alterations in hippocampal volume in schizophrenia (Narr et al, 2004), autism (Nicolson et al, 2006), mild cognitive impairment (Becker et al, 2006), dementia (Frisoni et al, 2006; Thompson et al, 2004a), and normal pediatric development (Gogtay et al, 2006), and has mapped the profile of anatomical changes in cohorts where differences in overall hippocampal volumes were not detectable (Nicolson et al, 2006).

In the current study, we hypothesized that unmedicated patients with bipolar disorder, relative to healthy comparison subjects, would exhibit localized alterations in hippocampal structure. Additionally, given prior evidence suggesting effects of lithium on brain structure (Manji et al, 2000b; Moore et al, 2000b; Sassi et al, 2002), we hypothesized that the pattern of hippocampal alteration would be different in lithium-treated relative to nonlithium-treated bipolar patients.

\section{METHODS}

\section{Participants}

This study was approved by the University of Pittsburgh biomedical institutional review board, and written informed consent was obtained from all subjects before participation, according to the Declaration of Helsinki. The sample included 33 patients with bipolar disorder and 62 healthy comparison subjects matched for age, sex, handedness, and level of education; a subset of this sample was previously reported in Sassi et al (2004) and Bearden et al (2007). Patients were recruited through the outpatient facilities of the University of Pittsburgh Medical Center, or through advertisements in the local media. The inclusion criteria were a DSM-IV diagnosis of bipolar disorder, subtype I or II, as determined by the Structured Clinical Interview for DSM-IV, Axis-I (First et al, 1996), in any mood state, age between 18 and 65 years, and, for the lithium-treated group, subjects had to be receiving lithium for at least 2 weeks before scanning. Subjects in the untreated group were required to be free of all psychotropic drugs for at least 2 weeks, and no lithium treatment for at least 1 month at the time of study participation. The rationale for this time frame is due to the elimination half-life for some antidepressants (such as fluoxetine, with a half-life time of 7-9 days). Although the elimination half-life of lithium averages $20-24 \mathrm{~h}$, it generally takes about 2 weeks for side effects to disappear completely. For this reason, we conservatively set the criterion at 1 month off of lithium before MRI scan (for the untreated group). Although the majority of subjects in the untreated group were not medication naïve (only two of the bipolar patients in this group had no prior history of any medication usage), most had been off all medications for much longer than 1 month (mean period off medication $=60.7$ weeks, \pm 35.8 weeks).

The Bech-Rafaelsen Mania Scale (Bech et al, 1979) and the Hamilton Depression Rating Scale (17-item version; Hamilton, 1960) were used to rate clinical symptoms and were administered within 1 week of the scan. All subjects had normal physical examination results and no history of neurologic problems. Exclusion criteria were any Axis-I comorbid psychiatric disorder, current medical problems, alcohol or substance abuse within 6 months preceding the study, or a lifetime history of any substance dependence. A total of eight bipolar subjects had a previous history of substance abuse occurring more than 6 months before the study (Table 1).

Healthy comparison subjects were recruited through local advertisements, according to the same exclusion criteria used for patients. Healthy control subjects had no DSM-IV Axis I disorders, as determined by the SCID-IV (First $e t$ al, 1996), no current medical problems, and no history of psychiatric disorders among first-degree relatives. The subject groups (healthy controls, $\mathrm{Li}+$, and $\mathrm{Li}-$ ) did not differ with regard to age, gender, race, or educational attainment (Table 1).

Bipolar patients were outpatients at time of assessment, with treatment histories of varying lengths (mean age of onset: $19.5 \pm 6.6$ years). The majority $(N=21,64 \%)$ was taking lithium at the time of evaluation, for a mean duration of 123 weeks ( \pm 226 weeks) (range 2-1000 weeks), at a mean dosage of $1125 \mathrm{mg} /$ day $( \pm 348.6$; range $675-2100 \mathrm{mg} /$ day). 
Table I Sample Demographics and Clinical Characteristics

\begin{tabular}{|c|c|c|c|c|}
\hline & $\begin{array}{l}\text { Lithium-treated patients with } \\
\text { bipolar disorder }(n=21)\end{array}$ & $\begin{array}{l}\text { Unmedicated patients with } \\
\text { bipolar disorder }^{a}(n=12)\end{array}$ & $\begin{array}{l}\text { Healthy comparison } \\
\text { subjects }(n=62)\end{array}$ & $\begin{array}{l}\text { Between group } \\
\text { differences }\end{array}$ \\
\hline Age (mean $\pm \mathrm{SD})$ & $32.9 \pm 11.4$ & $36.5 \pm 10.4$ & $32.7 \pm 10.1$ & $F(2,92)=0.69, p=0.51$ \\
\hline \% Right handed $(N)$ & $95 \%(20)$ & $92 \%(11)$ & $94 \%(58)$ & $\chi^{2}=0.17, p=0.92$ \\
\hline Education level (years) & $14.6 \pm 2.6$ & $15.4 \pm 3.4$ & $15.3 \pm 2.7$ & $F=0.56, p=0.57$ \\
\hline \multicolumn{5}{|l|}{ Race } \\
\hline \% Caucasian $(N)$ & $95 \%(20)$ & $92 \%(11)$ & $84 \%(52)$ & $\chi^{2}=4.34, p=0.36$ \\
\hline$\%$ African American $(N)$ & $5 \%(1)$ & 0 & 13\% (8) & \\
\hline$\%$ Other $(N)$ & 0 & $8 \%(1)$ & $3 \%(2)$ & \\
\hline \% Bipolar I disorder & $90 \%(19)$ & $58 \%(7)$ & & $\chi^{2}=4.72, p=0.07$ \\
\hline \% Bipolar II disorder & I0\% (2) & $42 \%(5)$ & NA & \\
\hline Age at onset & $18.2 \pm 5.9$ & $21.6 \pm 7.3$ & NA & $F=1.99, p=0.17$ \\
\hline Number of episodes & $19.7 \pm 26.4$ & $15.8 \pm 15.8$ & NA & $F=0.20, p=0.66$ \\
\hline Current mood state & & & & $\chi^{2}=5.30, p=0.052$ \\
\hline Depressed & 19\% (4) & $58 \%(7)$ & NA & \\
\hline Euthymic & $81 \%(17)$ & $42 \%(5)$ & & \\
\hline Past history of substance abuse ${ }^{b}$ & $33 \%(4)$ & $19 \%(4)$ & NA & $\chi^{2}=0.85, p=0.36$ \\
\hline
\end{tabular}

Mean \pm standard deviation for age and education levels are expressed in years; HDRS, total score on the 17-item Hamilton Depression Rating Scale.

an the unmedicated bipolar group, three subjects had previously received treatment with lithium, and three had previously taken valproate. Additional previous medications include: olanzapine $(n=1)$, tranylcypromine $(n=2)$, fluoxetine $(n=2)$, lorazepam $(n=1)$, imipramine $(n=1)$, citalopram $(n=2)$, clomipramine $(n=1)$, and levothyroxine $(n=1)$. Two subjects were medication-naïve.

$\mathrm{b}$ In the unmedicated bipolar group, two subjects had a past history of alcohol and cannabis abuse, one of alcohol abuse alone, and one had a past history of abuse of alcohol, cannabis, cocaine, and opioids. In the lithium-treated group, two subjects had a past history of alcohol abuse, one of alcohol and cannabis abuse, and one had a past history of cannabis abuse alone.

Mean blood level of lithium in the lithium-treated group was $0.79 \mathrm{mEq} / \mathrm{l}( \pm 0.29$; range $0.40-1.49 \mathrm{mEq} / \mathrm{l})$. In addition to lithium, two patients were also taking tranylcypromine $(10-20 \mathrm{mg})$, one was taking levothyroxine, and one was taking citalopram $60 \mathrm{mg}$ plus levothyroxine. The remaining treated patients $(N=17)$ received lithium monotherapy. Lithium-treated bipolar patients did not differ from unmedicated patients in duration of illness, age at onset, or number of previous episodes. However, there was a nonsignificant trend toward overrepresentation of bipolar II patients in the untreated group $(p=0.07)$, and depression severity scores were nonsignificantly higher in the untreated group ( $p=0.07$; Table 1$)$. All analyses were run with and without the seven Bipolar II subjects. Although $p$-values changed slightly, this did not affect whether each result was statistically significant, so we present results for the full sample.

\section{MRI Scanning}

Magnetic resonance imaging scans were acquired with a 1.5 T GE Signa Imaging System running Signa version 5.4.3 software. The scanning protocol was identical to that used in Sassi et al (2004) and Bearden et al (2007), and consisted of 124 contiguous $256 \times 192$ images acquired in the coronal plane, with acquisition parameters $\mathrm{TE}=5 \mathrm{~ms}, \mathrm{TR}=25 \mathrm{~ms}$, $\mathrm{FOV}=24 \mathrm{~cm}$, and slice thickness $=1.5 \mathrm{~mm}$. All MR images were processed with a series of manual and automated procedures developed at the UCLA Laboratory of Neuro Imaging that are described in detail in other reports (Thompson et al, 2004b), and summarized below.

\section{Anatomical Analysis}

MR images were resampled, using a trilinear interpolation, to have an in-plane resolution of $1 \times 1 \times 1 \mathrm{~mm}$, and then reoriented along the inter-hemispheric fissure in the axial and coronal views, and along the anterior-posterior commissure line in the sagittal view. The individual brain volumes were spatially realigned with the International Consortium for Brain Mapping nonlinear average brain template (ICBM152), using a six-parameter co-registration in the FLIRT program (available at http://www.fmrib.ox.ac.uk/fsl/). The hippocampi from each brain were then traced in native space using a software program (Tracer) (available at http:// www.loni.ucla.edu/Software/Software_Detail.jsp?software $\mathrm{id}=10$ ). This program allows the digitized surface contours to be displayed simultaneously in all three viewing planes to 
facilitate the accurate identification of neuroanatomic boundaries. Contours were drawn on images magnified four-fold to allow subvoxel precision and accurate tracking of small-scale features.

Hippocampi were manually traced bilaterally by a trained image analyst (M.P.), who was blind to all demographic variables, and had established excellent reliability with 'gold standard' ratings on a training set of six brains (intraclass correlation coefficient $\sim 0.90$ ). This level of agreement is comparable to that obtained in prior studies (Becker et al, 2006; Frisoni et al, 2006). Anatomical segmentation was performed using a standard neuroanatomic atlas of the hippocampus (Duvernoy, 1988) according to previously described criteria (Narr et al, 2004). Hippocampal models were delineated in contiguous coronal brain sections using standard guidelines (Pantel et al, 2005), including the hippocampus proper, dentate gyrus, and subiculum (Becker et al (2006) and Figure S1 for further details). Volumes obtained from these tracings were retained for statistical analyses.

Anatomical mesh modeling methods (Thompson et al, 1996) matched equivalent hippocampal surface points across subjects and groups (Thompson et al, 2004b). These methods reveal tissue alterations on the hippocampal surface, for example in regions approximately corresponding to the underlying cornu ammonis (CA) 1-3 subfields and subiculum/presubiculum (Figure 1a). To do this, a gridded surface is stretched over the hippocampus, using a rectilinear mesh of equally spaced 3-D points along the hippocampal axis and across the upper and lower surfaces. In the absence of any other landmarks to establish higherorder correspondences, mesh points with the same surfacebased coordinate are associated across subjects. These may or may not correspond exactly to the true cellular fields but are a reasonable approximation, and arguably the best possible approximation given the available landmarks in the data (Frisoni et al, 2006). The manually derived contours were made uniform by modeling them as a 3-D variable surface mesh (Figure 1b) (Thompson et al, 1996), allowing measurements to be made at corresponding surface locations in each subject. This procedure also allows the averaging of hippocampal surface morphological features across all individuals belonging to a group and records the amount of variation between corresponding surface points relative to the group averages. These methods are similar to the sampled medial description (M-rep) procedure (Styner et al, 2004) and high-dimensional computational mapping approach (Csernansky et al, 2002) developed for local shape analysis of the hippocampus.

To assess global hippocampal differences, the volumes of these 3-D models were measured in cubic millimeters. To measure local differences, a medial 3-D curve was derived from each individual's hippocampus threading down the central axis (Figure 1b). The distance of each surface point from this centerline measures the radial size of the hippocampus. Regressions were performed at each surface point to map linkages between radial size and covariates such as diagnosis and treatment group. In addition, we chose to examine hippocampal parameters both with and without brain size correction in our statistical analyses, given that relationships between hippocampal size and brain size may differ across
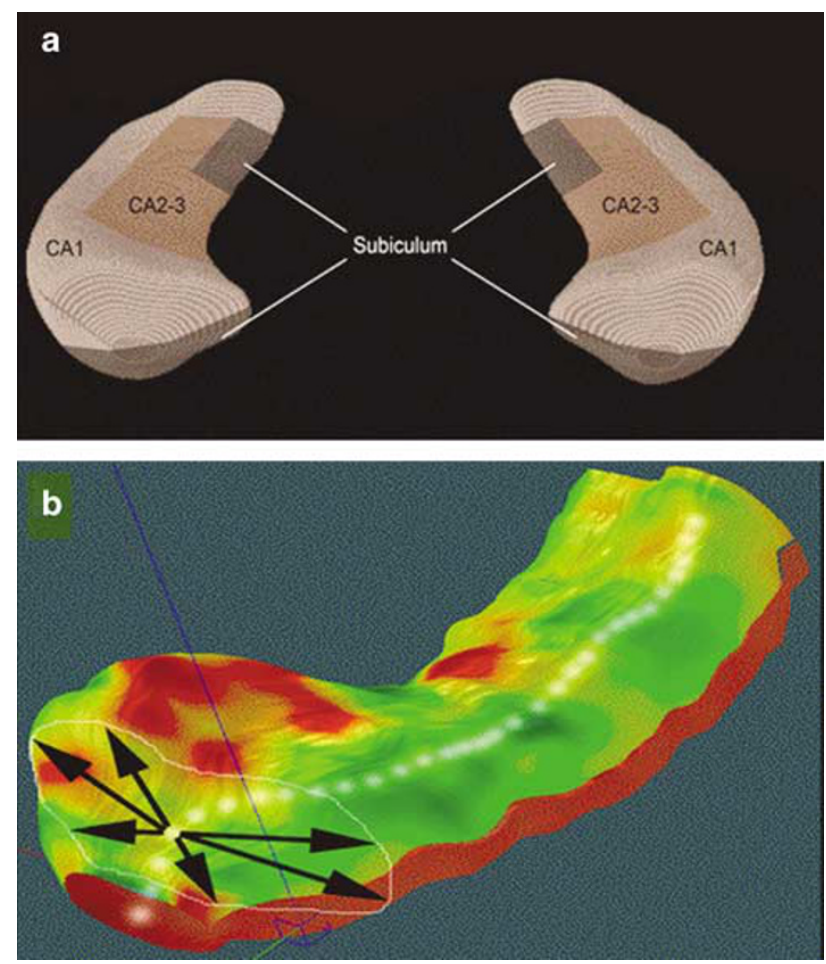

Figure I (a) Topographic correspondence of pathology on blank MR-based models of the hippocampal formation of normal controls. Based on Duvernoy (Duvernoy, 1988), where neuropathologic areas are shown in seven equally spaced coronal slices spanning the entire length of the hippocampus. CA, cornu ammonis. (b) Depicts the surface of the hippocampus, traced in consecutive coronal magnetic resonance image sections, which is composed of discrete triangular tiles that are spatially uniform and can be averaged across subjects. A 3-D medial curve is derived from each individual hippocampus (arrows in part b). The distance from this axis to the surface is the dependent variable in the regression analyses. See 'Methods' section of the text for details.

diagnostic groups, and our goal was to target differences specific to the hippocampus.

Given that independent statistical tests were made at many hippocampal surface points and adjacent data points are highly correlated, permutation testing was employed to control for multiple comparisons (Thompson et al, 2004b). For this purpose, hippocampal surfaces were randomly assigned to either patient or control groups 100000 times (while keeping the number of subjects in each group the same), and a new statistical test was performed at each hippocampal surface point for each random assignment. The number of significant results from these randomizations was then compared with the number of significant results in the true assignment to produce a corrected overall significance value for the uncorrected statistical maps. These permutations measure the distribution of features in the statistical maps that would be observed by accident if group assignment was random, and provide a $p$-value for the observed effects that is corrected for multiple comparisons. Permutation was conducted both for negative disease effects (CTL $>$ BPD) and for positive disease effects (BPD $>$ CTL), as well as for results in lithium-treated and non-lithium-treated groups. 


\section{RESULTS}

\section{Overall Volumetric Differences}

To provide context for the hippocampal maps, hippocampal volumes in patients with bipolar disorder, as compared to controls, are shown in Figure 2. At a global level, the total volume of the hippocampus differed significantly between controls and bipolar subjects $(\mathrm{F}(2,92)=6.72, p=0.002)$. The total volume of the hippocampus was significantly larger in the lithium-treated patients with BPD compared with the controls (by 10.3\%; least significant difference test, $p=0.001$; Cohen's $d=0.84)$ and compared with the untreated BPD patients (by 13.9\%; $p=0.003$; Cohen's $d=1.1$ ). In contrast, the total hippocampal volume of the untreated BPD patients did not significantly differ from that of controls $(3.1 \%$ smaller; $p=0.42$; but see maps, below, for more details). Lithium-treated BPD patients had significantly greater hippocampal volumes bilaterally than both the controls (least significant difference test, $p=0.005$ for left hippocampus, $p=0.002$ for right hippocampus) and the untreated BPD patients (left: $p=0.01$, right: $p=0.005$; Figure 2).

\section{3-D Hippocampal Maps}

Figure 3 reveals a pattern of localized deficit in the right hippocampus in unmedicated bipolar patients, as compared to healthy controls $(p=0.01$; Figure $3 \mathrm{a}$ and $c)$. Areas of

\section{Hippocampal Volumes in Lithium-Treated vs. Untreated Bipolar Patients and Control Subjects}

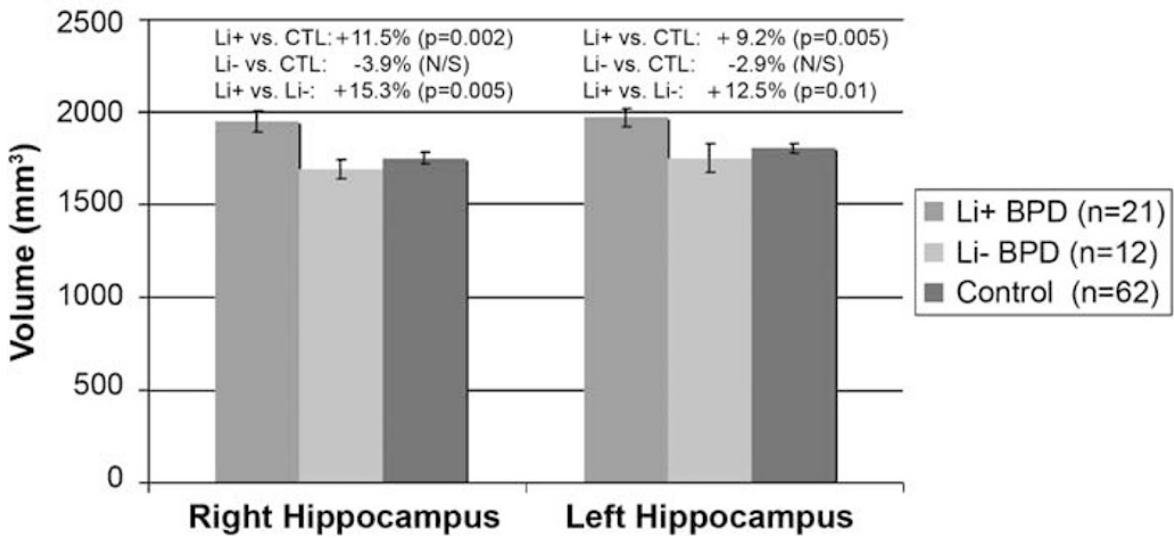

Figure 2 Hippocampal volumes. The mean \pm SEM for total volume of the right and left hippocampus in each subject group (lithium-treated bipolar patients, unmedicated bipolar patients, and healthy controls).
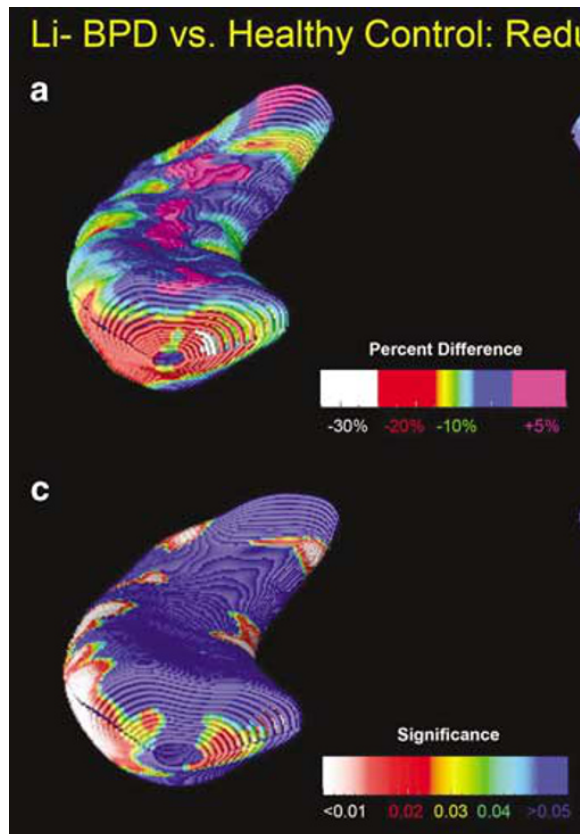

\section{Li+ BPD vs. Li- BPD: Reduction in Li- Group}

b
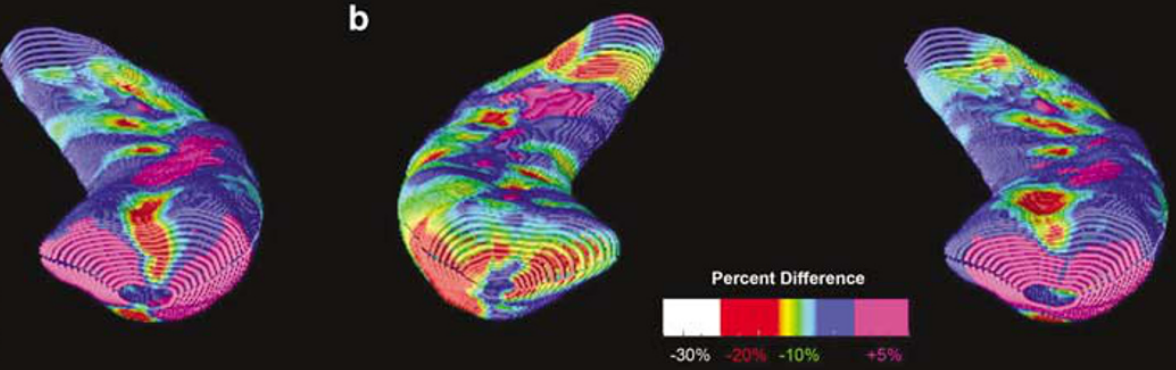

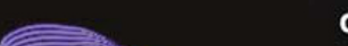

d
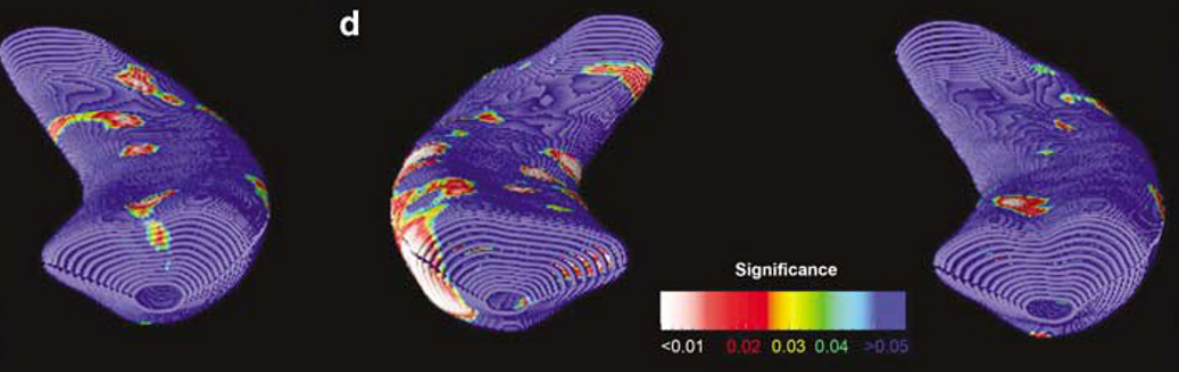

Figure 3 Statistical 3-D maps showing local differences in hippocampal structures between unmedicated patients with BP and control subjects, in terms of percent difference (a) and statistical significance (c). The pattern of findings is remarkably similar in unmedicated bipolar patients vs lithium-treated bipolar patients ( $b$ and $d)$. The right hippocampus is on the left side of the figure. 


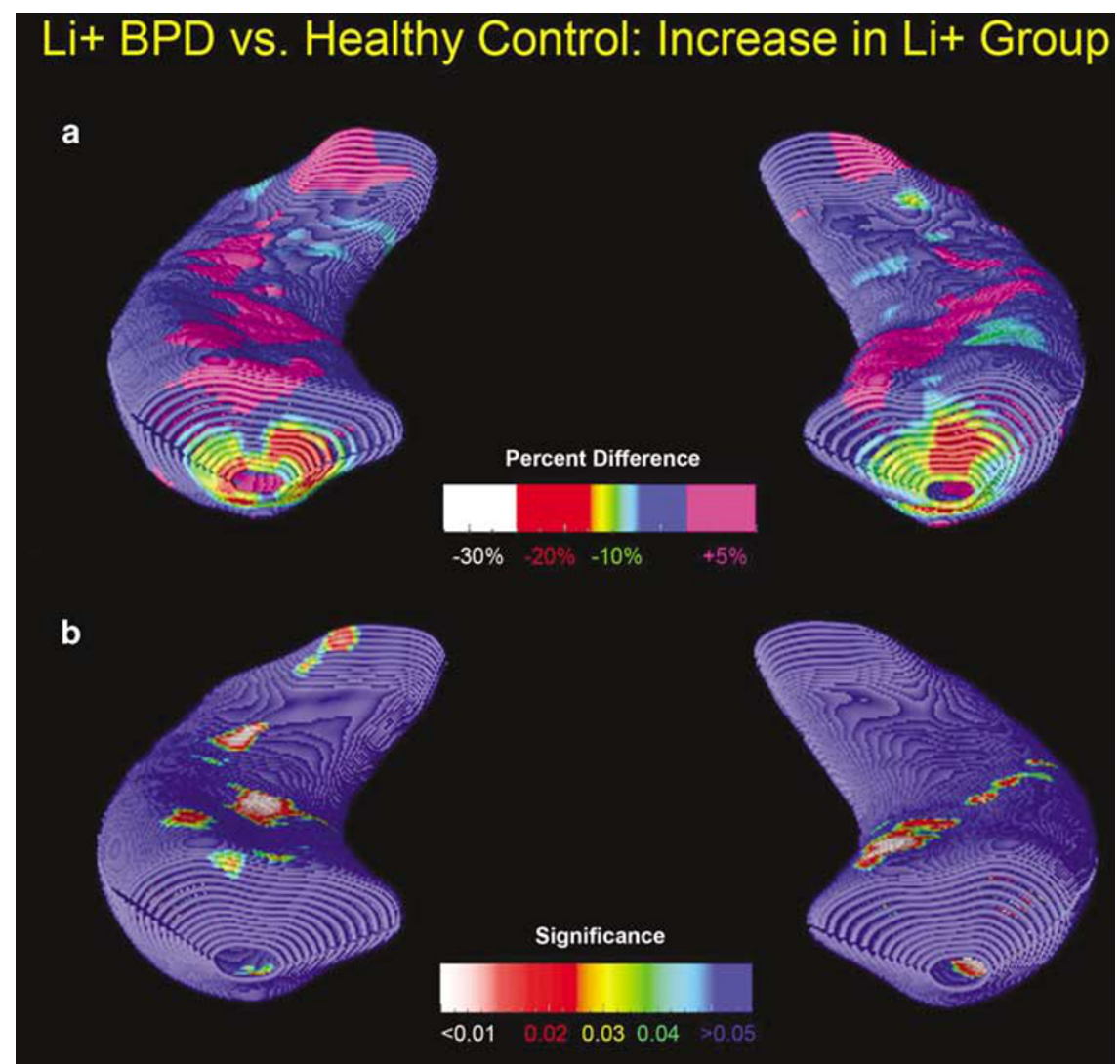

Figure 4 Statistical 3-D maps showing local differences in hippocampal structures between lithium-treated patients with BPD and control subjects, in terms of percent difference (a) and statistical significance (b). Again, the right hippocampus is on the left side of the figure.

significant hippocampal reduction in unmedicated patients were most prominent in regions corresponding to lateral CA1 subfields and subiculum (Figure 3). Maps for untreated patients did not significantly differ from controls on the left side $(p=0.13, \mathrm{NS})$. Unmedicated patients showed a highly similar pattern of deficit when compared to lithium-treated bipolar patients, in which areas of $10-20 \%$ reduction in the right hippocampus were observed in CA1 fields and parts of the subiculum (Figure $3 \mathrm{~b}$ and $\mathrm{d}$ ). Again, these group differences were significant following permutation testing for the right hippocampus $(p=0.03)$, but not for the left $(p=0.38$, NS). By contrast, localized hippocampal increases in lithium-treated BPD of $5 \%$ or greater (Figure $4 \mathrm{a}$ ) corresponded generally to a $p$-value $<0.05$ (Figure $4 \mathrm{~b}$ ) and mapped mainly to regions approximately corresponding to the CA1 subfields. Some small patches of deficit were observed in the subicular region in the tail of the hippocampus, but these did not reach statistical significance, as can be seen in Figure $4 \mathrm{~b}$.

\section{Correcting for Total Brain Volume}

Further, given that hippocampal volume and total brain volume are moderately correlated $(0.58$ across groups; $r=0.59(p<0.001)$ in controls, $r=0.28 \quad(p=0.38)$ in unmedicated patients, and $r=0.52(p=0.02)$ in lithiumtreated patients), we wished to examine the regional specificity of our hippocampal findings by controlling for total brain volume. These results indicated that, even when controlling for the highly significant effect of total brain volume on hippocampal volume $(\mathrm{F}(2,90)=38.67, p<0.001)$, group differences in hippocampal volume remained significant $(\mathrm{F}(2,90)=5.08, p=0.03)$.

\section{Effects of Other Clinical Variables}

Given that depression was nonsignificantly higher in the unmedicated group, analyses were redone with HDRS scores as a covariate. When controlling for depression severity, hippocampal volume still remained significantly different between lithium-treated and untreated patients $(\mathrm{F}(2,32)=5.35, p=0.01)$. The main effect of group $(\mathrm{Li}+v s$ $\mathrm{Li}-)$ remained highly significant $(\mathrm{F}(1,30)=4.19, p=0.05)$, while the effect of depression was not significant in the model $(\mathrm{F}(1,30)=1.39, p=0.25$, NS), indicating that the group differences were not accounted for by differences in level of depression.

In addition, although groups did not differ in terms of gender distribution, we conducted secondary analyses to control for any possible confounding effects of gender. Although there was a main effect of gender on total hippocampal volume $(\mathrm{F}(1,91)=5.92 ; p=0.017)$, hippocampal volume still remained significantly different between lithium-treated and untreated patients when covarying for gender $(\mathrm{F}(1,91)=6.31 ; p=0.003)$. Furthermore, when both gender and total brain volume were incorporated simultaneously in the model, the main effect of group remained significant $(\mathrm{F}(1,90)=2.45, p=0.03)$. 
Exploratory analyses were conducted to assess the effects of duration of lithium treatment and lithium dosage on hippocampal volume. As the duration of lithium treatment variable significantly departed from normality, it was normalized using a logarithm transformation. After normalization, there was not a significant linear correlation between duration of lithium use and hippocampal volume (left: $r=0.23, p=0.31$, NS; right: $r=-0.20, p=0.39$, NS). In addition, no significant relationships were observed between hippocampal volume and lithium dosage, nor lithium blood level within the lithium-treated group (all $p>0.10$ ). Additional analyses were conducted to assess (1) the potential confounding effects of other medications and a past history of comorbid substance abuse in the lithiumtreated group, (2) the potential confounding effect of the different proportions of bipolar I disorder $v s$ bipolar II disorder subjects in the unmedicated and lithium-treated groups, and (3) the effect of age at onset and duration of illness on hippocampal volume. Group comparisons were re-analyzed after excluding the four subjects on additional medications (tranylcypromine, levothyroxine, and citalopram) in the lithium-treated group. Group differences in hippocampal volume remained highly significant $(\mathrm{F}(2,88)=8.63, p \leqslant 0.001)$. Additionally, group differences in hippocampal volume remained highly significant after excluding the eight bipolar patients with a previous history of substance abuse from the analysis $(\mathrm{F}(2,84)=5.57$, $p=0.005)$, and after excluding the seven bipolar II patients from the analysis $(\mathrm{F}(2,85)=7.63, p \leqslant 0.001)$.

Finally, no significant correlations were detected between hippocampal volume and age at onset, number of prior episodes, or duration of illness ( $p>0.10$ for all comparisons).

\section{DISCUSSION}

These maps provide two novel findings regarding regional hippocampal alterations in bipolar disorder. Specifically, we observed localized hippocampal reductions in unmedicated bipolar patients, predominantly on the right, as compared with both normal controls and lithium-treated bipolar patients. In contrast, increased hippocampal volume was associated with lithium treatment in bipolar patients. Thus, the sensitive methods employed here suggest two possible explanations for the absence of observed differences in hippocampal volume in prior studies: (1) subtle, highly localized effects may not be detectable by standard volumetric methods, and/or (2) the effects of medication treatment may ameliorate or reverse pre-existing hippocampal pathology in bipolar disorder.

It is important to note that in this cross-sectional study, it cannot be ruled out that the observed group differences were present before initiation of lithium. However, it is tempting to speculate that these results may reflect postulated effects of neuropil increase related to lithium treatment, manifested as volumetric increases on MRI (Moore et al, 2000b). Further corroborating these data, in a prospective MRI study with healthy volunteers scanned both before and after 4 weeks of lithium treatment $(N=13)$, using voxel-based morphometry, we found significant increases in gray matter concentration in the left cingulate, left precuneus, and right superior frontal gyrus (Monkul et al, 2004). In addition, using cortical pattern matching methods to map gray matter concentration across the entire cortical surface, we found significant gray matter increases, particularly in the anterior cingulate and paralimbic cortices, in lithium-treated patients with bipolar disorder, as compared to healthy controls (Bearden et al, 2007).

Both overall volumetric results and 3-D radial distance maps indicated highly significant differences between lithium-treated and drug-free patients. Although global volumetric results did not significantly differ between unmedicated patients and controls, 3-D radial distance maps indicated localized hippocampal deficits in unmedicated patients, in regions approximately corresponding to CA1 subfields and parts of the subiculum, but no sign of reduction in regions corresponding to CA2 and CA3 subfields. Interestingly, this pattern is quite similar to that observed in other disorders with known hippocampal pathology, specifically Alzheimer's Disease and mild cognitive impairment, in which the CA1 subfields and subiculum have been shown to be differentially affected, in both in vivo neuroanatomic studies (Apostolova et al, 2006; Csernansky et al, 2005; Frisoni et al, 2006) and neuropathology studies (Van Hoesen and Hyman, 1990). Our functional MRI studies have demonstrated functional segregation of the human hippocampus during learning; specifically, regions corresponding to CA2 and CA3 fields are activated during storage, whereas those corresponding to the subiculum activate during retrieval of new information (Zeineh et al, 2003). Our neuroanatomic findings are thus compatible with prior neuropsychological findings indicating primary encoding and retrieval, rather than storage, deficits in patients with bipolar illness (Bearden et al, 2006). Further study of the relationship between functional and structural changes of the hippocampus is clearly warranted in future studies of bipolar disorder.

Our finding of more pronounced reduction in the right hippocampus in unmedicated patients is in line with evidence that bipolar disorder is more likely to be associated with right-side cerebral pathology; in cases of 'secondary mania' resulting from cerebral trauma, temporal lobe epilepsy, or stroke, most patients have lesions in righthemisphere limbic regions (Starkstein et al, 1991). Moreover, our findings are highly consistent with those of Hwang et al (2006), who recently reported significant shape differences in the right striatum in drug-naive bipolar patients relative to healthy comparison subjects, but found that drug-treated bipolar patients did not differ from controls. Our results indicate that this pattern extends to the hippocampal formation as well.

These findings add to a growing body of evidence for subtle neuroanatomic alteration in patients with bipolar disorder, and strongly suggest that drug treatment status is an important factor in assessing such differences in bipolar patients. In particular, these findings suggest a possible explanation for inconsistent findings across previous studies. Hippocampal differences may be somewhat localized, and difficult to detect in small, heterogeneous samples using global measures (McDonald et al, 2004). The identification of regional abnormalities in hippocampal structure may thus help to elucidate the underlying pathophysiological mechanisms associated with bipolar 
disorder, and also indicate functional systems that may be selectively disturbed in the illness.

The mechanism of lithium action is still largely unknown, but recent animal and human studies provide converging evidence for its potential neurotrophic effects (Manji et al, 2000a). At the molecular level, lithium has been shown to robustly increase levels of the neuroprotective protein B-cell lymphoma/leukemia-2 gene (bcl-2) in the hippocampus and frontal cortex and to promote neurite outgrowth (Chen et al, 1999), and may also mediate downstream effects of several neurotrophic factors (Manji et al, 2000b). In adult rodents, lithium produced a significant $25 \%$ increase in new cells in the dentate gyrus, demonstrating that this agent enhances hippocampal neurogenesis (Chen et al, 2000).

Although here we did not detect an association with dose or duration of lithium treatment on hippocampal anatomy, the relationship between duration of treatment and brain structural changes is likely to be complex and nonlinear, possibly involving an interaction between effects of lithium treatment and normal aging processes. Although one postmortem investigation noted reduction in non-pyramidal neurons in sector CA2 of the hippocampal formation in a small sample of bipolar patients with heterogeneous medication histories (Benes et al, 1998). Rajkowska (2000) observed that the thickness of sublayer IIIc in frontal cortex is greater and pyramidal cell density tends to be lower in subjects with a long exposure to lithium, suggesting that a compensatory increase in dendritic and/or glial neuropil, and consequent decrease in neuronal density, may be a response to long-term medication treatment. As such questions cannot be addressed in this naturalistic, crosssectional study, we are actively investigating neuroanatomic, as well as neurocognitive, effects of duration and dosage of lithium treatment in a prospective longitudinal study.

Certain limitations of the current study must be noted. As we did not specifically design this study to examine the effects of lithium treatment, we were unable to match the patient groups on all clinical variables; in particular, there was a nonsignificant trend toward higher depression severity scores in the untreated group at the time of scanning, and a trend toward overrepresentation of bipolar II patients in the untreated group. However, our results remained significant after controlling for the severity of depressive symptoms, and the results of our additional analyses specific to the bipolar I subgroup were almost identical to those obtained for the larger sample, suggesting that diagnostic heterogeneity was not a substantial confounding factor. Furthermore, prior studies have not reported structural anatomic differences as a function of current mood state (Brambilla et al, 2005), so we do not believe that this presents a significant confound. It should also be noted that, although the 12 patients in the untreated group were on no medications at the time of scanning, they were not necessarily medication-naïve. Indeed, three subjects in this group had previously received treatment with lithium, although two had been lithium-free for more than 2 years at the time of scanning, whereas the third had discontinued lithium 6 weeks before the MRI scan. In the context of this naturalistic study, we could not address the question of whether lithium's effects on hippocampal anatomy persist after the medication is discontinued, but this is a key question to investigate in future studies. Finally, our lithium-treated sample included patients who had been on lithium for varying time periods, and dosages were not uniform across subjects. In the context of a clinical trial, such variables could be better controlled. These limitations notwithstanding, this study is, to our knowledge, the first to describe differences in hippocampal size and morphology associated with lithium treatment in bipolar patients. As such, replication with a larger homogenous group of subjects may serve to confirm the results observed here.

In addition, although we cannot exclude the possibility that these findings are related to osmotic effects of lithium, leading to changes in water content in the brain, overall volumetric differences were specific to gray matter, with no change in white matter volume in lithium-treated patients (GM: $\quad \mathrm{F}(1,81)=10.42, \quad p=0.002 ; \quad \mathrm{WM}: \quad \mathrm{F}(1,81)=1.42$, $p=0.24$, NS). A purely osmotic action would be unlikely to be restricted to gray matter alone, which argues against this interpretation. Furthermore, Duning et al (2005) recently reported that severe dehydration (lack of fluid intake for $16 \mathrm{~h}$ ) - and subsequent rehydration - both resulted in a $<1 \%$ change in brain volume; given that we observed hippocampal gains more than 10 times that great in lithium-treated bipolar patients, osmotic effects are unlikely to account for our findings. Although another study recently found a mean elevation in frontal cortex tissue water in rats treated with lithium for 5 weeks (Phatak et al, 2006), there were no significant differences in percent tissue water between the lithium-fed rats and controls in the hippocampus. The mechanism for this change in frontal cortex was unclear, as it did not appear to result from changes in inositol concentration or blood sodium concentration. Given data suggesting that peripheral thyroid hormone levels may affect cerebral blood flow and glucose metabolism (Marangell et al, 1997), examination of thyroid function may also be important to consider in future studies.

Notably, other psychotropic agents, including fluoxetine (Santarelli et al, 2003) and atypical antipsychotics (Braus et al, 2001), may also affect neuronal viability. For example, in a recent clinical trial (Lieberman et al, 2005), patients treated with the typical antipsychotic agent haloperidol showed progressive gray matter loss, whereas olanzapinetreated patients did not, suggesting that olanzapine may be neuroprotective, less neurotoxic, or both (Molina et al, 2005). Using a mouse model, Santarelli et al (2003) find that by disrupting antidepressant-induced neurogenesis, the behavioral response to antidepressants is blocked, suggesting that the behavioral effects of chronic antidepressants may be mediated by the stimulation of neurogenesis in the hippocampus. Duman and Monteggia (2006) posit that decreased levels of brain-derived neurotrophic factors, as well as other neurotrophic factors, could contribute to the atrophy of certain brain structures, including the hippocampus and prefrontal cortex, that has been observed in depressed subjects. Nevertheless, although there is clear evidence that intervention with selective serotonin reuptake inhibitors can impact brain activation, as evidenced by functional neuroimaging and PET studies (see Linden (2006) for a review), to our knowledge, there are no published data on structural neuroanatomic changes, as a function of antidepressant use in humans. In any case, to 
address this possible confound, we conducted additional analyses excluding the four lithium-treated bipolar subjects who were taking adjunctive medications, and we find no difference in our original results.

These observations demonstrate the importance of taking medication effects into account when interpreting data from in vivo neuroimaging studies and post-mortem reports. Furthermore, other factors that may contribute to hippocampal alterations in bipolar patients, such as stressful life events, and duration and chronicity of illness, should be systematically examined in future investigations.

In conclusion, the sensitive 3-D mapping methods employed in this study were able to detect significant right-sided hippocampal reduction in unmedicated patients with bipolar disorder relative to both healthy controls and relative to lithium-treated patients. It is tempting to infer that the observed differences may suggest a mechanism of action for lithium's therapeutic effects, but these findings clearly need to be replicated in prospective longitudinal studies. The functional significance of these changes, with regard to their relationship to memory function in particular, is also an essential topic for future investigation.

\section{ACKNOWLEDGEMENTS}

This study was supported in part by Grants K23 MH07464401 (CEB), MH 01736, MH 30915, RR020571, Krus Endowed Chair in Psychiatry (UTHSCSA), Veterans Administration (VA Merit Review), NARSAD, CNPq (Conselho Nacional de Desenvolvimento Científico e Tecnológico, Brazil-no. 200006/04-5; to MP) and CAPES Foundation (Brazil). Algorithm development was supported by Grants from the National Institute for Biomedical Imaging and Bioengineering, the National Center for Research Resources, the National Institute on Aging, and the National Institute for Child Health and Human Development (EB01651, RR019771, AG016570, HD050735 to PMT).

\section{DISCLOSURES}

The authors have no conflicts of interest and no relevant financial disclosures to declare.

\section{REFERENCES}

Altshuler LL, Ventura J, van Gorp WG, Green MF, Theberge DC, Mintz J (2004). Neurocognitive function in clinically stable men with bipolar I disorder or schizophrenia and normal control subjects. Biol Psychiatry 56: 560-569.

Apostolova LG, Dutton RA, Dinov ID, Hayashi KM, Toga AW, Cummings JL et al (2006). Conversion of mild cognitive impairment to Alzheimer disease predicted by hippocampal atrophy maps. Arch Neurol 63: 693-699.

Bearden CE, Glahn DC, Monkul ES, Barrett J, Najt P, Kaur S et al (2006). Sources of declarative memory impairment in bipolar disorder: mnemonic processes and clinical features. J Psychiatr Res 40: 47-58.

Bearden CE, Thompson PM, Dalwani M, Hayashi KM, Lee AD, Nicoletti $M$ et al (2007). Greater cortical gray matter density in lithium-treated patients with bipolar disorder. Biol Psychiatry 62: 7-16.
Bech P, Bolwig TG, Kramp P, Rafaelsen OJ (1979). The BechRafaelsen mania scale and the hamilton depression scale. Acta Psychiatr Scand 59: 420-430.

Becker JT, Davis SW, Hayashi KM, Meltzer CC, Toga AW, Lopez OL et al (2006). Three-dimensional patterns of hippocampal atrophy in mild cognitive impairment. Arch Neurol 63: 97-101.

Benes FM, Kwok EW, Vincent SL, Todtenkopf MS (1998). A reduction of nonpyramidal cells in sector CA2 of schizophrenics and manic depressives. Biol Psychiatry 44: 88-97.

Brambilla P, Glahn DC, Balestrieri M, Soares JC (2005). Magnetic resonance findings in bipolar disorder. Psychiatr Clin North Am 28: 443-467.

Braus DF, Ende G, Weber-Fahr W, Demirakca T, Henn FA (2001). Favorable effect on neuronal viability in the anterior cingulate gyrus due to long-term treatment with atypical antipsychotics: an MRSI study. Pharmacopsychiatry 34: 251-253.

Chen G, Rajkowska G, Du F, Seraji-Bozorgzad N, Manji HK (2000). Enhancement of hippocampal neurogenesis by lithium. $J$ Neurochem 75: 1729-1734.

Chen G, Zeng WZ, Yuan PX, Huang LD, Jiang YM, Zhao ZH et al (1999). The mood-stabilizing agents lithium and valproate robustly increase the levels of the neuroprotective protein bcl-2 in the CNS. J Neurochem 72: 879-882.

Csernansky JG, Wang L, Jones D, Rastogi-Cruz D, Posener JA, Heydebrand G et al (2002). Hippocampal deformities in schizophrenia characterized by high dimensional brain mapping. Am J Psychiatry 159: 2000-2006.

Csernansky JG, Wang L, Swank J, Miller JP, Gado M, McKeel D et al (2005). Preclinical detection of Alzheimer's disease: hippocampal shape and volume predict dementia onset in the elderly. Neuroimage 25: 783-792.

Duman RS, Monteggia LM (2006). A neurotrophic model for stress-related mood disorders. Biol Psychiatry 59: 1116-1127.

Duning T, Kloska S, Steinstrater O, Kugel H, Heindel W, Knecht S (2005). Dehydration confounds the assessment of brain atrophy. Neurology 64: 548-550.

Duvernoy H (1988). The Human Hippocampus: An Atlas of Applied Anatomy. JF Bergman Verlag: Munich, Germany.

Eichenbaum H, Fortin NJ (2005). Bridging the gap between brain and behavior: cognitive and neural mechanisms of episodic memory. J Exp Anal Behav 84: 619-629.

First MB, Spitzer RL, Gibbon M, Williams JBW (1996). Structured Clinical Interview for DSM-IV Axis I Disorders, Research Version, Patient Edition. Biometrics Research Department, New York State Psychiatric Institute: New York.

Frisoni GB, Sabattoli F, Lee AD, Dutton RA, Toga AW, Thompson $\mathrm{PM}$ (2006). In vivo neuropathology of the hippocampal formation in AD: A radial mapping MR-based study. Neuroimage 32: 104-110.

Gogtay N, Nugent III TF, Herman DH, Ordonez A, Greenstein D, Hayashi KM et al (2006). Dynamic mapping of normal human hippocampal development. Hippocampus 16: 664-672.

Gourovitch ML, Torrey EF, Gold JM, Randolph C, Weinberger DR, Goldberg TE (1999). Neuropsychological performance of monozygotic twins discordant for bipolar disorder. Biol Psychiatry 45: 639-646.

Hamilton M (1960). A rating scale for depression. J Neurol Neurosurg Psychiatry 23: 56-62.

Hwang J, Lyoo IK, Dager SR, Friedman SD, Oh JS, Lee JY et al (2006). Basal ganglia shape alterations in bipolar disorder. Am J Psychiatry 163: 276-285.

Kempermann G (2002). Regulation of adult hippocampal neurogenesis - implications for novel theories of major depression. Bipolar Disord 4: 17-33.

Kieseppa T, Tuulio-Henriksson A, Haukka J, Van Erp T, Glahn D, Cannon TD et al (2005). Memory and verbal learning functions in twins with bipolar-I disorder, and the role of informationprocessing speed. Psychol Med 35: 205-215. 
Lieberman JA, Tollefson GD, Charles C, Zipursky R, Sharma T, Kahn RS et al (2005). Antipsychotic drug effects on brain morphology in first-episode psychosis. Arch Gen Psychiatry 62: 361-370.

Linden DE (2006). How psychotherapy changes the brain-the contribution of functional neuroimaging. Mol Psychiatry 11: 528-538.

Manji HK, Moore GJ, Chen G (2000a). Clinical and preclinical evidence for the neurotrophic effects of mood stabilizers: implications for the pathophysiology and treatment of manicdepressive illness. Biol Psychiatry 48: 740-754.

Manji HK, Moore GJ, Chen G (2000b). Lithium up-regulates the cytoprotective protein $\mathrm{Bcl}-2$ in the $\mathrm{CNS}$ in vivo: a role for neurotrophic and neuroprotective effects in manic depressive illness. J Clin Psychiatry 61(Suppl 9): 82-96.

Marangell LB, Ketter TA, George MS, Pazzaglia PJ, Callahan AM, Parekh $\mathrm{P}$ et al (1997). Inverse relationship of peripheral thyrotropin-stimulating hormone levels to brain activity in mood disorders. Am J Psychiatry 154: 224-230.

McDonald C, Zanelli J, Rabe-Hesketh S, Ellison-Wright I, Sham P, Kalidindi S et al (2004). Meta-analysis of magnetic resonance imaging brain morphometry studies in bipolar disorder. Biol Psychiatry 56: 411-417.

Molina V, Reig S, Sanz J, Palomo T, Benito C, Sanchez J et al (2005). Increase in gray matter and decrease in white matter volumes in the cortex during treatment with atypical neuroleptics in schizophrenia. Schizophr Res 80: 61-71.

Monkul E, Dalwani M, Nicoletti M, Soares J (2004). Brain gray matter changes after lithium treatment: a voxel-based morphometry study in healthy individuals. Bio Psychiatry 55: 202S.

Moore GJ, Bebchuk JM, Hasanat K, Chen G, Seraji-Bozorgzad N, Wilds IB et al (2000a). Lithium increases $\mathrm{N}$-acetyl-aspartate in the human brain: in vivo evidence in support of bcl-2's neurotrophic effects? Biol Psychiatry 48: 1-8.

Moore GJ, Bebchuk JM, Wilds IB, Chen G, Manji HK (2000b). Lithium-induced increase in human brain grey matter. Lancet 356: $1241-1242$.

Narr KL, Thompson PM, Szeszko P, Robinson D, Jang S, Woods RP et al (2004). Regional specificity of hippocampal volume reductions in first-episode schizophrenia. Neuroimage 21: 1563-1575.

Nicolson R, DeVito TJ, Vidal CN, Sui Y, Hayashi KM, Drost DJ et al (2006). Detection and mapping of hippocampal abnormalities in autism. Psychiatry Neuroimaging Res 148: 11-21.

Pantel J, Cretsinger K, Keefe H (2005). Hippocampus tracing guidelines. Available at http://www.psychiatry.uiowa.edu/mhcrc/ pdf/papers/hippocampus.pdf. (Accessed November 1, 2005).

Phatak P, Shaldivin A, King LS, Shapiro P, Regenold WT (2006). Lithium and inositol: effects on brain water homeostasis in the rat. Psychopharmacology (Berlin) 186: 41-47.
Rajkowska G (2000). Postmortem studies in mood disorders indicate altered numbers of neurons and glial cells. Biol Psychiatry 48: 766-777.

Santarelli L, Saxe M, Gross C, Surget A, Battaglia F, Dulawa S et al (2003). Requirement of hippocampal neurogenesis for the behavioral effects of antidepressants. Science 301: 805-809.

Sassi RB, Brambilla P, Hatch JP, Nicoletti MA, Mallinger AG, Frank $\mathrm{E}$ et al (2004). Reduced left anterior cingulate volumes in untreated bipolar patients. Biol Psychiatry 56: 467-475.

Sassi RB, Nicoletti M, Brambilla P, Mallinger AG, Frank E, Kupfer DJ et al (2002). Increased gray matter volume in lithium-treated bipolar disorder patients. Neurosci Lett 329: 243-245.

Silverstone $\mathrm{PH}, \mathrm{Wu} \mathrm{RH}$, O'Donnell T, Ulrich M, Asghar SJ, Hanstock CC (2003). Chronic treatment with lithium, but not sodium valproate, increases cortical $\mathrm{N}$-acetyl-aspartate concentrations in euthymic bipolar patients. Int Clin Psychopharmacol 18: 73-79.

Starkstein SE, Fedoroff P, Berthier ML, Robinson RG (1991). Manic-depressive and pure manic states after brain lesions. Biol Psychiatry 29: 149-158.

Styner M, Lieberman JA, Pantazis D, Gerig G (2004). Boundary and medial shape analysis of the hippocampus in schizophrenia. Med Image Anal 8: 197-203.

Thompson PM, Hayashi KM, De Zubicaray GI, Janke AL, Rose SE, Semple J et al (2004a). Mapping hippocampal and ventricular change in Alzheimer disease. Neuroimage 22: 1754-1766.

Thompson PM, Hayashi KM, Sowell ER, Gogtay N, Giedd JN, Rapoport JL et al (2004b). Mapping cortical change in Alzheimer's disease, brain development, and schizophrenia. Neuroimage 23(suppl 1): S2-S18.

Thompson PM, Schwartz C, Toga AW (1996). High-resolution random mesh algorithms for creating a probabilistic 3D surface atlas of the human brain. Neuroimage 3: 19-34.

van Gorp WG, Altshuler L, Theberge DC, Mintz J (1999). Declarative and procedural memory in bipolar disorder. Biol Psychiatry 46: 525-531.

Van Hoesen GW, Hyman BT (1990). Hippocampal formation: anatomy and the patterns of pathology in Alzheimer's disease. Prog Brain Res 83: 445-457.

Videbech P, Ravnkilde B (2004). Hippocampal volume and depression: a meta-analysis of MRI studies. Am J Psychiatry 161: $1957-1966$.

Wright IC, Rabe-Hesketh S, Woodruff PW, David AS, Murray RM, Bullmore ET (2000). Meta-analysis of regional brain volumes in schizophrenia. Am J Psychiatry 157: 16-25.

Zeineh MM, Engel SA, Thompson PM, Bookheimer SY (2003). Dynamics of the hippocampus during encoding and retrieval of face-name pairs. Science 299: 577-580.

Supplementary Information accompanies the paper on the Neuropsychopharmacology website (http://www.nature.com/ npp). 